Results 90/147 and 132/166 of patients enrolled onto "UgWHO03" and "WHO07" respectively and were able to provide adequate sputum for culture. For UgWHO03: five cases of smear negative PTB and four cases of smear positive PTB diagnosed by algorithm and three cases by culture. For WHO07: 18 cases of smear negative PTB and seven cases of smear positive PTB diagnosed by algorithm and 1 case by culture. Sensitivity for smear-negative PTB increased nonsignificantly from $62.5 \%$ to $94.7 \% \quad(p=0.065)$, specificity was maintained ( $99 \%$ vs $98 \%$, not significant).

Conclusion Sample size was limited by the ability to provide sputum but there was a non-significant trend towards increased sensitivity which approached significance. This suggests that in the context of a more rapid pathway this algorithm may be beneficial.

\section{P2-485 IMMUNOGENICITY OF PANDEMIC INFLUENZA A (H1N1) MONOVALENT VACCINE IN PATIENTS ON LONG-TERM HAEMODIALYSIS}

doi:10.1136/jech.2011.142976m.12
Methods This study incorporates a review and meta-analysis of the literature on the relationship between temperature and morbidity. Utilising a number of different electronic databases, a comprehensive search was performed, the results of which were filtered against pre-defined selection criteria. Subgroups were defined according to type of morbidity and whether a heat or cold effect was examined. The studies were combined using both fixed and random effects models to examine both heterogeneity and the effect of a unit change in temperature.

Results 37 studies were selected to be included in the review. Studies only listing correlations were analysed separately from those reporting actual responses in morbidity per unit change in temperature. In particular, for hot effects an increase in $1^{\circ} \mathrm{C}$ resulted in an increased risk of morbidity (RR: $1.002,95 \%$ CI 1.001 to 1.003), and similarly for a $1^{\circ} \mathrm{C}$ decrease in temperature for cold effects $(R R$ : $1.003,95 \%$ CI 1.002 to 1.005 ).

Conclusion This study provides a thorough survey of the literature relating to the effects of temperature on morbidity. The mean effects of temperature were found from relevant studies, along with an examination of important subgroups.

\section{P2-487 POPULATION CHANGES, SOCIAL DISPARITIES AND CHILD HEALTH IN LATIN AMERICA}

doi:10.1136/jech.2011.142976m.14

Introduction Influenza is a potentially serious infection among haemodialysis (HD) patients, and the antibody response to vaccines among HD patients is thought to be weaker than that in healthy people. We conducted this study for the purpose of evaluating the immunogenicity and reactogenicity of the pandemic vaccine among patients on long-term HD.

Materials and Methods During the 2009-2010 influenza season, 170 HD patients (108 men, 62 women; mean age: $62.2 \pm 13.2$ years, mean and max duration of HD: 12.3 and 35 years, respectively) received two doses of monovalent inactivated unadjuvanted vaccine. Immunogenicity of the vaccine was evaluated according to conventionally used international criteria (EMEA, 1997; FDA, 2007). Results The geometric mean of HI titre 4 weeks after vaccination increased from a pre-vac level of 7.0 to 32.5 for the first injection, and to 37.8 for the second injection. The sero-conversion rate was $48 \%$ and sero-protection rate was $54 \%$ for the first injection, and $53 \%$ and $57 \%$ respectively for the second injection. The occurrence of systemic rush was significantly higher compared with healthy adults.

Conclusions A two-shot influenza vaccination series showed a booster effect and achieved the EMEA criteria at the first vaccination. No serious adverse events were detected, but systemic rush occurred frequently. A Dysfunction in detoxification ability due to the patients' renal disease may have caused this.

Funding This study was supported by a research grant for Research on Emerging and Re-emerging Infectious Diseases, Ministry of Health, Labor and Welfare, Japan.

\section{P2-486 ENVIRONMENTAL TEMPERATURE VARIATION AND MORBIDITY: A META-ANALYSIS}

doi:10.1136/jech.2011.142976m.13

L Turner, ${ }^{*}$ S Tong. Queensland University of Technology, Brisbane, Queensland, Australia

Introduction The relationship between temperature and mortality has been well documented in the literature, with studies examining the effects of both temperature variations and extremes. The impact of temperature on morbidity however has received far less attention. In particular, to date there has been no study of the existing literature examining the effect temperature has on morbidity.

M G Vega-Lopez, ${ }^{*}$ G J Gonzalez-Perez, P P Quintero-Vega. University of Guadalajara, Guadalajara, Mexico

Introduction America Latina -a region known for its social disparities- is currently experiencing a profound demographic and epidemiological transition. In such sense, this paper seeks to analyse the relationship between social inequalities, demographic changes and child health in Latin America.

Methods Information about population and health was obtained from diverse official sources; different statistics like ratios among extreme socioeconomic groups or countries (ordered in quartiles according to Human Development Index 2007) were calculated to quantify the difference in health indicators among countries or in selected countries.

Results Despite the observed population ageing in recent years, this study reveals that the population under 15 years old in Latin America in 2030 will be only slightly lower to the existent in 1990; furthermore, child population's will be increased in poorest countries of the region. Data reflects large differences in health indicators: in 2008 , infant mortality rate in quartile of the poorest countries almost triplicate the rate of the richest quartile; inside each studied country, notables differences related with poverty are also observed: in all the cases, the infant malnutrition rate or the $<5$ mortality rate are much higher in poorest population's quintile that in the richest quintile.

Conclusions The above-mentioned aspects evidence a dramatic inequity in child health -related to social disparities-, something more relevant since in absolute terms, Latin American population $<15$ will maintain similar levels in next decades and that in the poorest countries -or in the poorest regions in each country-child population's will be higher.

\section{P2-488 DENTAL CARIES IN A PORTUGUESE SAMPLE OF SCHOOLCHILDREN}

doi:10.1136/jech.2011.142976m.15

N Veiga, 0 Amaral, J Pereira, C Pereira.* Cl \& DETS, Health School, Polytechnic Institute of Viseu, Viseu, Portugal

Background Dental caries is the most prevalent disease worldwide. The objective of this study was to determine the mean of decayed/ 